\title{
Les ingrédients des fétiches
}

The Essential Ingredients of Fetishes

\section{Albert de Surgy}

\section{OpenEdition}

Journals

Édition électronique

URL : http://journals.openedition.org/span/1323

DOI : $10.4000 /$ span. 1323

ISSN : 2268-1558

\section{Éditeur}

École pratique des hautes études. Sciences humaines

\section{Édition imprimée}

Date de publication : 1 novembre 1993

Pagination : 103-143.

ISSN : 0294-7080

\section{Référence électronique}

Albert de Surgy, "Les ingrédients des fétiches », Systèmes de pensée en Afrique noire [En ligne],

12 | 1993, mis en ligne le 08 janvier 2014, consulté le 01 mai 2019. URL : http:// journals.openedition.org/span/1323 ; DOI : 10.4000/span.1323 


\section{LES INGRÉDIENTS DES FÉTICHES}

\section{par}

\section{Albert de Surgy}

Les objets de culte évhé pouvant prétendre à l'appellation de fétiches se répartissent en deux grandes classes: les bo servant à provoquer des effets ponctuels, immédiats, par enchantement des esprits qui participent à l'engendrement des phénomènes, et les vodu achetables, adoptés non seulement pour bénéficier d'une direction et d'une protection plus suivie, mais surtout de forces spirituelles et d'événements heureux (de Surgy, 1990). Aucune frontière infranchissable ne sépare toutefois les uns et les autres et ils se trouvent constitués selon les mêmes principes, en se fondant sur les propriétés subtiles d'ingrédients matériels utilisés par ailleurs dans de simples buts thérapeutiques.

Quels sont ces ingrédients? Que représentent-ils? A quoi exactement servent-ils? Les instruments de puissance qu'ils permettent de composer different-ils radicalement des objets d'adoration mieux connus, tels que statues, icônes, diagrammes rituels, corps vivants..., qui se donnent à contempler et se trouvent souvent complétés par des autels? Les pratiques qui s'y rapportent sont-elles spécifiques de l'aire culturelle considérée ou peut-on en rencontrer de semblables ailleurs, non seulement en Afrique mais aussi dans d'autres continents et jusque

Fétiches II. Puissance des objets, charme des mots, systèmes de pensée en Afrique noire, 12, 1993 
dans les sociétés techniquement les plus avancées? Telles sont les questions auxquelles je vais m'efforeer ci-dessous de répondre.

\section{Ces ingrédients ne se donnent pas à voir}

Dans l'introduction de son ouvrage Heviesso et le bon ordre du monde (1987), Bruno Gilli affirme nettement que: "Le vodu n'est pas ce qu'on voit et ce qui apparaît, il est essentiellement ce qu'on ne voit pas et ce qui n'apparaît pas. Le vodu n'est pas la réalité matérielle en tant que telle, mais plutôt la force ou la puissance qui se manifeste en elle et par elle". Cette affirmation, que ne contesterait aucun de mes propres informateurs, se traduit dans le fait que les éléments essentiels d'un fétiche sont toujours enfouis sous quelque chose ou dans quelque chose. Ce qui s'en laisse voir n'est jamais qu'un revêtement ou une partie subsidiaire permettant de les localiser et de les identifier.

Considérons le cas très simple de deux plantes dotées de vertus magiques:

L'une permet d'échapper aux accidents ou de s'en tirer sans grand dommage. Elle suscite une atmosphère spirituelle défavorable à la réalisation de phénomènes violents.

La seconde permet de ne pas être atteint par des balles de fusil, des coups de couteau, des coups de matraque ou des jets de pierre. Son interaction avec l'esprit de son porteur crée autour de ce dernier une atmosphère qui fait hésiter un agresseur et lui fait rater son attaque.

Pour ne pas qu'elles soient détériorées et réduites en miettes, il est nécessaire de les protéger dans un emballage. Cet emballage devra permettre de les distinguer l'une de l'autre. On le fabriquera par exemple en tissu rouge pour la première et en tissu blanc pour la seconde ${ }^{1}$.

Le cas se complique dès qu'un sujet devient propriétaire d'un grand nombre d'objets de ce genre. Il lui faut graver ou fixer sur leur

${ }^{1} \mathrm{C}$ 'est ainsi enveloppées qu'elles me furent amicalement remises pour me souhaiter bon voyage. 
enveloppe des tracés ou des éléments signifiants adéquats. L’apparence ainsi conférée à l'objet ne manque pas d'avoir quelque rapport avec sa constitution et sa fonction. Cependant l'objet ne lui doit en rien sa puissance. Les supports matériels de ses vertus se voient seulement affublés ainsi d'une figure permettant commodément de le reconnaître. Cette figure pourrait fort bien être remplacée par un numéro de code. Lui attribuer une importance excessive, demeurer fasciné par elle, conduit à se fourvoyer aussi gravement que si l'on voulait étudier les propriétés et l'usage de médicaments sans en faire la moindre analyse chimique, en ne prenant en considération que les mots et les graphismes inscrits sur les boîtes dans lesquels ils se trouvent livrés, en ne les jugeant que d'après l'image de marque qui en résulte.

Cette observation concernant de simples plantes vaut a fortiori pour les bo et les vodu. Leur efficacité repose sur des ingrédients, présentés comme indispensables à leur fabrication, qui sont systématiquement protégés des regards en même temps que d'une détérioration par un contact étranger. Ces ingrédients, principalement végétaux, peuvent être insérés dans une statuette ou un instrument rituel, ou être attachés à eux une fois convenablement enveloppés, ou être simplement enfermés dans une poterie ou tout autre récipient (calebasse, bouteille). Dans le cas des vodu, ils sont le plus souvent enterrés sous un objet figuratif en bois, en terre pétrie ou en ciment, ou sous un vulgaire monticule portant à son sommet ou sur ses flancs des décorations caractéristiques. De telles représentations et décorations sont certes plus ou moins soignées, plus ou moins belles ou impressionnantes. Cependant, elles ne sont jamais considérées comme essentielles. Certains féticheurs, et parmi les plus grands qui ne se soucient guère d'impressionner une clientèle, les suppriment tout bonnement ou les réduisent à un repérage des plus discrets de ce qu'ils ont enfoui dans le sol de leur cour ou de leur maison et qu'un visiteur non averti ne remarquera même pas $^{2}$.

\footnotetext{
2 Pour avoir le plus de chance de l'emporter sur les autres, mieux vaut, estiment-ils, leur laisser ignorer que l'on est puissant. Seuls les prétentieux ou les étourdis se mettent volontiers en avant et se font en conséquence plus facilement abattre.
} 
Parmi mes photographies de fétiches, celle qui a le plus frappé mon entourage fait apparaître une machine à coudre dressée sur un socle de ciment en compagnie de restes d'une serrure et d'un piège, portant encore trace de libations et d'offrandes de nourriture. Voilà, dirait-on, une belle composition surréaliste rapprochant de manière surprenante divers objets s'ignorant habituellement les uns les autres. En brisant nos habitudes perceptives, ne nous ouvre-t-elle pas l'esprit à l'étrange, au mystère, au sacré ?

Dans une composition de ce genre transparaît peut-être en effet un souci de ne pas arrêter le regard sur une forme de signification précise. Il n'en reste pas moins que ce qui importe à son propriétaire est l'informe force qu'il y juge attachée, matériellement évoquée par les ingrédients enfouis à sa base. En l'occurrence il s'agit d'un bo-vodu (vodu de simple protection) appelé Gu, travaillant avec la même énergie spirituelle que le vodu du métal de même nom. Pour l'identifier, il est d'usage de déposer au sommet de la butte (aujourd'hui cimentée) qui en indique l'emplacement un ou plusieurs objets de métal. Ce sont aussi bien, et done de préférence, de vieux objets cassés ou hors d'usage qui, au lieu d'être jetés à la poubelle, trouvent là une plus honorable fin. N'importe lesquels peuvent convenir vieux couteaux, houes fendues, vieux boulons, vieilles pièces de mécanique..., et pourquoi pas vieille machine à écrire ou vieille machine à coudre? Il en existait une dans la maison, abandonnée par une femme. On trouva donc astucieux de la planter là.

La plupart du temps, au lieu d'un objet placé clairement en évidence comme celui-là, c'est tout un fatras apparemment hétéroclite d'objets que l'on trouve déposés sur un vodu, accrochés à lui, incrustés dans ses flancs ou abandonnés au sol contre lui. Il en résulte parfois un certain effet artistique. Le féticheur ne se prive pas en effet de jouer avec tout cela pour le disposer ou le redisposer à sa convenance du moment. L'esthétique correspondante est propre aux fétiches et mérite d'être étudiée pour elle même (comme le fait notre collègue Michèle Coquet), mais ne nous renseigne guere sur ce qu'ils sont.

Apparaissent ainsi livrés à notre regard:

- des accessoires rituels (couteaux, clochettes, cannes, verres, écuelles...), 
- des éléments décoratifs en rapport avec le caractère ou le type d'action du fétiche (collier de coquilles d'escargots, lunettes de soleil, symboles de fer forgé...).

- des restes d'offrandes sacrificielles.

- des restes de produits ayant été employés ou fabriqués lors de précédentes cérémonies. Au cas où ils pourraient resservir (médicaments, poudres, pierres...), ou leurs contenants être réutilisés, ils ne sont pas jetés.

- des objets abandonnés à titre d'ex-voto par des malades guéris (chapeau, sandalettes, sac de voyage...).

Pour qui connaît le vodu et les pratiques qui s'y rapportent, de tels éléments ont tous quelque rapport avec lui. Ils ne sont donc pas aussi hétéroclites qu'on est enclin à l'estimer de prime abord. Ils constituent une sorte de parure, agencée selon un certain goût, vouée à ne pas trouver d'unité autrement qu'en référence à ce qu'elle contribue à dissimuler.

Quand je parle d'ingrédients des fétiches, je ne désigne aucune de ces choses accumulées sur lui ou contre lui en raison d'une certaine affinité avec lui et permettant sans hésitation de l'identifier. Je prends en considération celles qui en constituent profondément la substance même ou le noyau et correspondent au type de force dont on entend qu'il fasse preuve.

\section{Ils ne renvoient pas à des esprits}

Il s'agit principalement de feuilles qui, du fait qu'on tient surtout compte de leurs vertus occultes, sont appelées ama et non plus $g b e^{3}$.

${ }^{3}$ Gbe désigne l'herbe ou la végétation à l'état brut, telle qu'elle est utilisée par des guérisseurs appelés atikèwsla (celui qui prépare des médicaments) ou gbesala (celui qui arrache des herbes). Ama désigne les mêmes éléments végétaux tels qu'ils sont utilisés par des spécialistes appelés amawsla ou amadhala (celui qui prépare ou qui cuisine des $a m a$ ), respectant avant tout leur valeur symbolique et le "souffle" ( $g$ bogbo) qui y demeure attaché. "En brousse on l'appelle gbe. A la maison (rapportée là pour un usage magique) on l'appelle ama", affirment-ils fréquemment en commençant à préparer leurs décoctions ou leurs poudres magiques. 
Mais nous y trouvons encore des éléments minéraux, animaux, humains et artisanaux, ainsi que des témoins de toutes sortes d'événements que, par extension, on se permet aussi d'appeler ama.

Comme toutes les créatures vivantes, les végétaux sont dotés d'un esprit appelé luvho, mot désignant l'ombre portée au sol qui flanque tout ce qui est mis en relief sur la terre. Un tel esprit assure la transposition en réalités objectives, sensibles, des ordres créateurs ou impulsions à réaliser quelque chose qui lui parviennent depuis la portion de l'univers des possibilités au service de laquelle Dieu l'a affecté. L'homme a seulement ceci de particulier qu'il possede deux esprits: un "esprit de vie" (agbe luvhs), responsable de son existence corporelle, et un "esprit de mort" ( $k u$-luvhs), responsable de son existence mentale invisible à autrui, dont l'appariement constitue ce que je nomme son âme.

Il arrive qu'en collectant des feuilles, et éventuellement de l'écorce ou des morceaux de racines, on s'adresse nommément à l'esprit de la plante concernée pour s'excuser d'avoir à lui dérober des fragments du corps qui lui appartient. On lui offre à cette occasion quelques pièces de monnaie ou des cauris, un peu de boisson, parfois même un ouf ou un petit poulet. Cependant ce n'est pas lui que l'on ramène à la maison et ce qui lui a été extorqué ne servira jamais à établir ensuite un quelconque rapport avec lui.

Il en va de même avec les autres catégories d'ingrédients. Aucun d'eux n'est mis a profit pour entrer en rapport avec l'esprit dans le domaine de résidence ou d'activité duquel ils ont été prélevés. En particulier aucun fragment de corps humain, de vêtement ou d'attribut de fonction n'est jamais utilisé comme relais de communication avec l'esprit de celui qui en fut ou qui en demeure propriétaire ${ }^{4}$. Cela est fort bien mis en évidence par les deux façons opposées de traiter des choses ayant appartenu à un "mauvais mort" selon qu'on cherche à

4 Il peut arriver qu'un envoûtement soit facilité par des objets ayant été en contact intime avec la personne visée, mais nous verrons qu'ils ne permettent pas d'entrer en communication "sympathique" avec elle. Ils servent à évoquer le type d'énergie spirituelle dont elle est approvisionnée pour lui couper à la source cette énergie ou introduire en elle des intentions qui lui sont étrangères. 
attirer et honorer celui-ci sur une sorte d'autel, ou bien seulement à fabriquer un fétiche.

Une personne sérieusement dérangée par un défunt ayant été prématurément privé de la vie dans des conditions tragiques et ne pouvant rejoindre en conséquence le pays de ses ancêtres est en effet orientée par un devin vers l'une des deux solutions suivantes:

- soit aménager (provisoirement) en brousse un lieu appelé tsinsphe où il viendra boire et recevoir de menues satisfactions, ou mieux encore le recueillir chez soi en lui installant quelque part une sorte d'habitacle où on lui fera périodiquement des offrandes, le prendre ainsi en considération, l'apaiser, le dorloter, l'aider à traverser l'épreuve qu'il subit et profiter, en compensation, de ses capacités de vision dans l'au-delà comme de gardiennage spirituel de la cour d'habitation. Il est toujours alors invité à décliner son identité et à préciser ses désirs.

- soit, en raison de son comportement vicieux ou agressif, ne pas faire acte de compassion envers lui, mais lui témoigner un total mépris, ne pas même chercher la plupart du temps à connaître son identité, le renvoyer comme un malpropre ou le ligoter pour l'empêcher de nuire. A cet effet on utilise un fétiche.

Dans le premier cas un grand respect est témoigné au disparu. De menus objets ayant jadis été en contact intime avec lui ou représentant son principe vital (perles, bracelets...) tiennent lieu de reliques aidant à le joindre, induisant sa présence et étendant à l'espace environnant l'influence bénéfique qu'il peut dispenser lui-même ou en vertu de son intégration à l'entourage d'une divinité.

Dans le second cas il est traité en importun ou en ennemi et ne bénéficie d'aucune considération. Le fétiche agit à son égard catégoriquement, tel un automate remettant à sa place ou renversant aveuglément dans le fossé tout ce qui gêne la progression de celui qui le contrôle.

Prenons deux exemples celui du vodu Gidiglo Adzamapho, qui agit fermement en douceur, et celui d'un bo Sibisaba qui est impitoyable à l'égard de l'esprit auquel il s'oppose.

Gidiglo Adzamapho permet de guérir les fous dont l'état est dû, comme il arrive souvent, à une aliénation par des âmes errantes de 
"mauvais morts". Les esprits d'un petit chien et d'un chat, séparément emballés vivants dans du tissu blanc et enterrés à l'emplacement du vodu, associés aux esprits d'un pigeon noir et d'un pigeon blanc, enfouis vivants de leur côté à l'intérieur de deux idoles de terre pétrie disposées sur une étagère dressée par dessus, sont envoyés se mettre respectivement en rapport avec l'esprit de mort ( $k u$-luvho) et l'esprit de vie (agbe-luvhs) de l'âme qui tourmente le malade pour les persuader ou les contraindre de s'en écarter. Le fou subit ensuite une purification en brousse, par lavage avec une eau où trempent les feuilles du vodu puis par inoculation sous sa peau et par absorption d'ingrédients carbonisés réduits en poudre. Une fois guéri il est amené au cimetière des "mauvais morts" et y est de nouveau lavé après que l'on ait égorgé un coq sur sa tête pour achever de le dissocier de la compagnie des "mauvais morts" et les remercier d'avoir bien voulu le laisser tranquille.

Sibisaba exerce aussi bien une action offensive que défensive.

- D'une part, il permet d'ordonner à des âmes de "mauvais morts" d'aller déranger ou influencer l'esprit d'une personne vivante. A cet effet il ne travaille pas avec une âme définie qui serait fixée sur lui et a laquelle une sorte de culte serait rendu. Il donne pouvoir de forcer la première qui se présente à accomplir un travail dont elle est capable. Quiconque dispose d'un tel pouvoir n'a nul besoin de recourir toujours ensuite à celle qu'il a initialement soumise, et encore moins de s'en encombrer en la tenant emprisonnée quelque part à sa disposition. A supposer que cela finisse par être le cas, l'âme asservie n'est pas respectueusement traitée en personne défunte comme un forçat qu'il suffit de contraindre par l'intermédiaire d'un garde-chiourme, elle n'est même pas nommée.

- D'autre part il permet d'écarter radicalement d'une personne vivante l'âme d'un "mauvais mort" qui le dérange, sans entamer la moindre négociation avec elle. Après avoir mis son fétiche en activité par les paroles adéquates, le féticheur entraîne le malade en brousse, l'introduit dans une cavité, le rase, égorge sur lui un coq ou un cochon, puis le lave avec l'eau du pot rouge où trempent les herbes du bo et lui introduit un peu de poudre magique noire correspondante dans de petites incisions pratiquées sur la peau de son crâne comme au niveau 
de toutes ses articulations. Cette purification et cette fortification de ses défenses suffisent en principe à débarrasser le malade du mort qui le dérangeait. S'il y a lieu, c'est par un tout autre moyen qu'on cherchera à connaître l'identité de ce dernier, quelles étaient ses motivations et ce qu'il convient de faire pour l'apaiser.

Dans la confection d'un habitacle d'âme errante et dans celle d'un fétiche se manifestent deux attitudes opposées à l'égard des âmes dont le deuil n'a pas été parfaitement résolu. La première témoigne d'une profonde affectation de la personne par l'une de ces âmes. La seconde d'une superbe indifférence à leur égard.

Un autel de mauvais mort comporte habituellement un objet entier, au minimum une perle, symbolisant le type de puissance vitale du mort (puisque précisément destiné à fixer son agbe luvho ou esprit vital) ou quelque chose l'ayant profondément concerné, mais en aucun cas des morceaux de son cadavre. Parmi les ingrédients constitutifs des fétiches, en revanche, il n'est pas rare de rencontrer des restes d'un cadavre ou des choses demeurant fortement imprégnées par les effluves ou "saletés" de la mort. Il ne s'agit pas là d'éléments dignes d'être pieusement conservés en souvenir d'un décédé. Ils ont été arrachés, fragmentés, sans respect pour lui ou pour sa famille, et sans la moindre intention de conserver quelque rapport avec lui.

La confection de Gidiglo Adzamapho, déjà cité, nécessite une bande de pagne blanc ayant été offert pour enterrer un cadavre et des restes de feuilles ayant servi, le septième jour qui suit l'enterrement d'un mauvais mort, à purifier ceux qui ont manipulé son cadavre.

La confection de Sibisaba nécessite des herbes ayant été ramassées sur la tombe d'un bon mort et sur la tombe d'un mauvais mort.

Voici quelques autres exemples:

La confection d'Agbagli, qui permet aussi bien de tourner en sa faveur l'esprit d'une femme ou de son patron que de rendre quelqu'un malade ou de le faire mourir, nécessite notamment:

- un tibia de mauvais mort et un tibia de bon mort,

- une éponge ayant servi à laver un mort,

- une bande de tissu ayant servi à masquer la bouche et le nez d'un mort, 
- un morceau de pagne et des cheveux d'un mauvais mort,

- enfin un petit cercueil dans lequel le fétiche est fabriqué.

La confection d'Abrayiboe, qui permet d'évoquer une personne dans un miroir pour l'influencer ou la faire mourir, nécessite notamment:

- de la percale blanche et de la percale noire ayant été enterrées avec un cadavre,

- des cheveux d'un mauvais mort.

La confection de Sokpata, qui permet de tuer en mettant en jeu la puissance du vodu de la foudre, nécessite notamment:

- un tibia humain de mauvais mort,

- vingt et une dents de mauvais mort,

- un morceau de tissu qu'un mauvais mort portait avant de mourir, - des herbes arrachées au dessus de la tombe d'un mauvais mort, - un morceau de racine ayant été tranchée en creusant une tombe.

On aura compris que, pour infliger la mort à quelqu'un ou le protéger de la mort, il convient d'inclure dans le fétiche, non seulement des signes de mort, mais des restes de morts ou d'objets spirituellement contaminés par un mort. Je dis restes car ces ingrédients ne sont pas vénérés comme reliques d'un mort particulier; ils sont traités en choses permettant seulement d'évoquer la puissance de réalisation ou de propagation d'un certain type de malheur par lequel il a été frappé.

Tous les fétiches, loin de là, ne sont pas construits dans ce but. Cependant, à examiner de près ce dont ils se composent, il apparaît que ce sont essentiellement des restes de corps vivants, d'objets ou d'événements recherchés pour autant qu'ils conservent l'empreinte des forces ayant contribue à leur donner forme.

Indépendamment d'éventuels restes humains, nous y trouvons des restes d'animaux petits animaux entiers desséchés, morceaux de peau ou griffes d'animaux féroces, têtes de serpents, coquilles d'escargots, morceaux de termitières ou de nids de fourmis arboricoles, plumes de certains oiseaux, moustaches ou poils de certains animaux, notamment de l'écureuil fouisseur de savane, etc. Tous sont encore jugés imprégnés de la vertu de l'animal correspondant ou de l'une de ses activités. 
Ils ne sont jamais utilisés pour commander un travail à son propre esprit désincarné.

Outre des restes d'animaux, nous y trouvons bien entendu des restes végétaux, surtout des feuilles, mais aussi des racines, des écorces, des morceaux de branches, non pas traités en représentants de plantes particulières, mais en vecteurs des vertus attachées aux espèces d'où ils proviennent ou au type d'accident les ayant affectés (barrer ou longer un chemin, avoir poussé à l'emplacement d'une tombe ou entre les rails du chemin de fer, etc.).

Nous y trouvons encore des restes d'objets ayant subi l'influence de certains corps ou de certaines activités (morceaux de vêtements portés par une évocatrice des défunts, poussière ou détritus du marché, etc.), ou ayant été fabriqués et de préférence utilisés par des vivants dans une intention précise (cadenas, serrures, hameçons, fusil miniature ayant été forgé à partir d'un morceau de vrai fusil cassé, morceau de corde ayant servi à attacher les bagages sur la galerie d'un minibus). Quand ils n'ont pas subi l'influence d'une creature vivante, ils peuvent avoir subi celle d'une divinité (morceau de rideau d'une porte de sanctuaire) ou celle d'une âme errante ou d'un esprit de la nature ayant résidé à leur emplacement ou à proximité immédiate. Ceux qui ne paraissent marqués par personne ni par aucun événement singulier, n'en sont pas moins jugés avoir été imprégnés par un certain souffle terrestre (pierre latéritique, concrétion blanche trouvée par des paysans à l'intérieur de la terre, particules luisantes dégagées du sol apres une averse) et sont traités en restes d'un processus physique ayant été dirigé ou influencé lui aussi par des agents spirituels.

Un féticheur les considere comme des matériaux qui, après avoir été d'une façon ou d'une autre en relation avec des esprits, ont été abandonnés par ces esprits et se prêtent maintenant à être manipulés en ne tenant compte que de leurs subtiles propriétés objectives.

\section{Ils ne renvoient pas à autre chose}

Les fétiches ne sont pas destinés à représenter quelque chose d'autre. Seul leur enrobage ou l'objet auquel ils sont accrochés nous 
en signifie les principaux caractères et usages possibles. Leur partie essentielle et cachée n'est qu'un magma d'ingrédients pouvant difficilement être considéré comme un objet. Il s'agit là d'une chose mystérieuse n'ayant aucune prétention figurative, même dans les cas où un ou plusieurs éléments présentant une forme intelligible y sont inclus.

Entassés les uns sur les autres, rassemblés en paquets, ces ingrédients amalgament leurs vertus. Ils ne sont pas articulés les uns aux autres comme des segments de discours et ne représentent pas non plus, dans leur ensemble, une constellation de signifiés. Ils n'ont pas été rassemblés pour évoquer des principes, des valeurs et des distinctions intellectuelles. Ils ne se rapportent à aucun référent précis et ne possèdent aucune valeur sémantique. Ils ne nous disent rien. Tout simplement ils sont là.

Aucun d'eux ne nous renvoie à la totalité d'où il a été extrait ou à l'esprit ayant été responsable de son existence. Il ne sert pas davantage à évoquer une idée présentant un rapport naturel ou conventionnel avec lui. En persistant à vouloir les interpréter en ce sens, on en est arrivé à inventer à leur propos la malheureuse expression de signifiant flottant (qu'est-ce qu'une signification nous laissant dans l'ignorance de ce qu'elle signifie ?) ou à les présenter comme des éléments de l'indicible contexte, préverbal, subverbal ou paraverbal, qui supporte ou accompagne le contenu explicite du discours. Cela ne nous libère malheureusement pas d'une problématique de transposition discursive de quelque chose d'autre et ne conduit qu'à des théories insatisfaisantes. Essayons donc plutôt de nous fonder sur les conceptions en vigueur au sein même des populations concernées.

Selon le système du monde servant de cadre aux comportements magico-religieux dans l'aire culturelle adja-évhé, une signification résulte d'un rapport générateur entre le monde de la vie sur terre (kodzogbe), celui des formes matérialisées ou réalisées, et un monde des entrailles de la Terre (l'amedzophe ou "monde de l'origine") depuis lequel tout est envoyé prendre naissance, où est préalablement conçu ce que réussissent plus ou moins bien à objectiver les opérations souvent conflictuelles d'une multitude d'esprits. Une existence est ainsi une contribution singulière à la manifestation d'un univers déjà préfiguré quelque part. Elle a pour but de transposer en productions maté- 
rielles, culturelles ou institutionnelles le fruit d'un choix prénatal de possibilités de réalisation.

Un tel choix est définitif. Il nous est présenté comme la résultante d'une série de comportements librement décidés ayant porté à conséquence quelque part. Contrairement à ce qu'il en est pour des actions ordinaires, on n'en trouve sur terre aucune trace objectivement accessible. Il demeure enregistré dans la mystérieuse substance d'un invisible monde intérieur au monde, directement fécondé comme une matrice féminine par des germes d'origine céleste, se laissant assimiler en raison de ce contact immédiat avec le ciel à une sorte de terre céleste.

De même que toute réalisation doit être en continuité avec les réalisations passées, toute nouvelle conception doit être en continuité avec des conceptions précédentes. Le "monde de l'origine" n'est donc pas une sorte d'entrepôt d'archétypes éternels. Il est soumis à une évolution qui dépend non seulement de la façon dont les âmes y composent leurs programmes prénataux, mais aussi de la façon dont par la suite elles le cultivent, c'est-à-dire de la façon dont les germes d'événements qu'il recèle vont porter fruit sur terre avant de lui être restitués à l'état de nouveaux germes atténués ou renforcés. Tout en se présentant comme un monde de l'antériorité, il n'en appartient donc pas moins à l'empire de la Nature. Tout s'y transforme parallelement à ce qui n'en est jamais, à la surface de celle-ci, qu'une transposition au grand jour.

On l'imagine situé sous nos pieds, dans l'obscurité des profondeurs. Cependant ce qui en provient est jugé en sortir, à l'est, par l'abîme d'où le soleil paraît émerger chaque matin, et ce qui y fait retour est jugé y revenir, à l'ouest, par l'abîme à travers lequel le soleil paraît regagner chaque soir sa demeure. Les rapports entre les réalisations effectives et leurs référents intelligibles nous apparaissent ainsi étalés à l'horizontale dans une direction est-ouest. 
Cet axe longitudinal est parcouru par une énergie d'avènement, de soutènement et d'évacuation après achèvement ${ }^{5}$ des phénomènes. Une telle énergie assure la communication entre les entrailles de la génitrice universelle et la surface de son corps où vient se fixer ce dont elle a accouché. Elle correspond au dynamisme par lequel la nature ne cesse de faire passer les possibilités dont elle est grosse de la puissance à l'acte, d'assurer la gestation de toute production objective, de la maintenir un certain temps en état, puis de la désagréger pour qu'elle cède sa place à une autre. Elle est assimilée à un "souffle" cosmique véhiculant à destination des paroles créatrices émises par la "bouche" de la terre $^{6}$. Il s'agit là d'une invisible substance dynamique dont bénéficie tout ce qui existe. Or précisément les ingrédients d'un fétiche sont choisis en fonction du "souffle" (appelé gbogbo, littéralement respiration, ce qui s'exhale et s'inhale) qui leur est attaché.

Il est implicitement postulé que tout ce qui a été modelé, structuré ou organisé en requérant un certain type de force créatrice ou réalisatrice conserve d'une certaine manière l'empreinte de celle-ci et demeure en affinité avec elle. Il modifie automatiquement en lui et autour de lui le "souffle" cosmique en y reconstituant une image de la configuration des "souffles" ayant déterminé ce qu'il est devenu. II paraît ainsi exercer de l'attraction sur les énergies efficientes qui lui correspondent et cela explique qu'un souffle subtil paraisse lui rester associé alors même que, soustrait à un corps vivant ou produit par un corps vivant, il a objectivement perdu la vie ou du moins perdu contact avec elle.

${ }^{5}$ Du point de vue correspondant, ce qui est pleinement réalisé, ce qui a produit tous ses fruits, n'est plus sustenté par une telle énergie et n'est donc plus vivant. Ses constituants spirituels sont emportés par l'ouest pour être recyclés dans les entrailles de la terre.

${ }^{6}$ Ces paroles mettent un certain temps (analogue à celui de la gestation du fotus humain) à être acheminées jusque sur terre. Néanmoins un devin, par une sorte de téléphonie sans fil (mettant en jeu un fétiche) ou avec fil (mettant en jeu une âme d'ancêtre), peut entrer en communication immédiate avec la bouche d'où elles sortent pour lui extorquer, non pas cette fois des événements, mais des informations relatives à son état et à son activité. 
Remarquons à ce propos qu'il n'est pas d'objet concevable indépendamment d'un esprit qui le prend en charge pour le fabriquer, le modifier, l'utiliser dans une certaine intention, lui conférer tout simplement un certain sens ou en remanier le sens. Or une telle prise en charge ne s'effectue qu'en référence à un contenu du monde de l'origine, c'est-à-dire à un certain objet secret du désir. Que l'objet réel soit objectivement modifié ou qu'il en soit fait seulement une autre lecture, engage l'esprit de la même manière vis-à-vis de lui par un acte créateur de signification. Autrement dit la vertu exploitable de tout ingrédient magique, tout en lui étant objectivement liée, n'est pas indépendante de la grille d'interprétation mentale inculquée à son manipulateur par la tradition lignagère ou savante à laquelle il se rattache.

$\mathrm{Du}$ fait que cet ingrédient n'a vu le jour qu'en relation avec le monde de l'origine et a pu entrer en interaction importante avec plusieurs esprits, il est évident qu'il n'a pas perdu tout rapport avec des significations. Il reste d'ailleurs parfois signifiant. Néanmoins nous ne le trouvons pas utilisé dans un but sémantique, comme évocateur de l'un des fondements de notre compréhension du monde, mais pour servir de support ou de caisse de résonance à un certain type de souffle vital animant l'énonciation (créatrice ou purement verbale) de quelque chose. Pareil souffle n'est pas tenu prisonnier dans les limites des corps qu'il fait subsister, mais s'y introduit et en est exhalé. Il peut donc contaminer des objets ou des esprits voisins et déploie spontanément une influence analogue à celle des talismans.

Bref, un fétiche ne doit pas son efficacité à ce qu'il désigne ou aux significations qu'il est capable d'induire, mais à l'amalgame des $g b\lrcorner g b o$ de ses constituants, c'est-à-dire à une certaine formule concrète d'énergie vitale. Une simple fraction d'objet ou de corps convient donc parfaitement à l'usage qu'on veut en faire. De même qu'une courte portion de texte ou de partition musicale permet, quel qu'en soit le contenu, de remonter au type de sensibilité de son auteur (on reconnaît aussitôt du Proust, du Zola, du Mozart, etc.), un morceau de production naturelle (organique ou inorganique), artisanale ou culturelle suffit 
à donner à qui la recherche l'intuition du type de détermination mentale et de poussée créatrice auquel l'existence de ce à quoi il se trouvait intégré est imputable.

\section{Ils servent à entrer en rapport avec des puissances surnaturelles}

Ne renvoyant ni à des esprits ni à autre chose, les ingrédients des fétiches possèdent une puissance propre dont tirent directement profit les spécialistes appelés amats (propriétaires d'ama) ou amadhala (préparateurs d'ama) qui bornent leur ambition à manipuler habilement les influences.

Cette puissance ne dérive pas des propriétés physico-chimiques de leur substance mais de leur imprégnation par des forces ayant dû être exercées pour imposer une signification aux ensembles dont ils proviennent ${ }^{7}$. Elle est donc liée au potentiel d'intelligibilité qui leur a été conféré par une série de pressions spirituelles.

Un fétiche n'en est pas moins irréductible aux ingrédients qui le caractérisent. Il est le fruit d'un traitement rituel qui métamorphose ceux-ci en moyens d'accès au monde surnaturel. Ce qui le différencie radicalement d'un simple paquet, d'une simple décoction ou d'une simple poudre magique est qu'il fait l'objet de sacrifices dédiés à une puissance indépendante de sa matérialité tangible ou subtile $e^{8}$. A mon avis, on ne saurait toutefois le présenter comme un simple autel de cette puissance étant donné sa relation extrêmement forte avec elle. Il en constitue une sorte de prolongement artificiel ou de représentant

\footnotetext{
${ }^{7}$ Je rappelle que, du point de vue évhé, l'existence de toute chose est sensée car elle résulte d'un travail de transposition concrète d'une forme intelligible (fruit d'une conception opérée dans les entrailles de la terre) assumé par des esprits (luvho) humains ou non humains.

${ }^{8}$ De même, chez les Igala du Nigéria, les fétiches ode (les équivalents des bo évhé), dont l'univers recoupe celui des ebo (divinités équivalentes aux vodu évhé), sont composés par un rassemblement d'ingrédients identiques à ceux des médecines ogwu (les équivalents des ama évhé), mais, à la différence de ces dernières, reçoivent des sacrifices et mettent en rapport avec des puissances spirituelles spécifiques (J. Boston, 1971).
} 
sensible dans le monde. $Y$ toucher revient à tirer sur une sorte de lien sacré en déclenchant inévitablement une réaction à son autre bout.

a) Premièrement, alors que tous les fragments ou restes de corps dégagent du "souffle" (gbっgbs), on ne peut inventer de fétiche en composant grâce à eux, selon son goût, une formule pneumatique de son choix. Ou bien il faut aller en acquérir un auprès de quelqu'un qui en possède déjà un exemplaire. Ou bien il faut en recevoir la révélation par un de ses ancêtres, le plus souvent son ancêtre tutélaire (amedzots), ou par un génie de brousse (aziza ou gbetsage). De plus l'acquéreur ou le fondateur doit non seulement être instruit de sa composition matérielle, de la façon de l'entretenir, de ses interdits et des paroles avec lesquelles il convient de s'adresser à lui, mais surtout être mis rituellement en contact avec la puissance qu'il concerne.

De même que nul ne peut deviner seul ce qu'un mot isolé d'une langue inconnue signifie, nul ne peut découvrir seul, au simple examen d'un objet ou d'un ensemble d'objets, quelle est la puissance vers laquelle il est capable de conduire. Cela doit lui être révélé par une autre personne, morte ou vivante, se trouvant en contact avec elle, ou par un médiateur spirituel averti.

De même que toute combinaison de sons ne fait pas un mot, toute combinaison de choses ne fait pas un fétiche.

De même que toute émission de sons produit physiquement de l'effet sur ses auditeurs, toute combinaison de choses exerce de l'influence sur les créatures qui s'en approchent, mais cela indépendamment de tout rapport avec une puissance surnaturelle. On aura beau lui parler et immoler dessus un poulet ou un pigeon, ce sera en vain on ne fera au mieux que fortifier les vertus qu'elle possede en tant qu'ama ${ }^{9}$. On ne la transformera ni en bo, ni en vodu.

\footnotetext{
${ }^{9}$ Notons que personne ne s'avise de s'unir sacrificiellement à des ama qui ne sont pas ceux d'un bo ou d'un vodu. Une telle pratique serait très risquée car elle mettrait le sujet en condition d'être envahi par des esprits inférieurs sans aucun contrôle surnaturel. Des immolations de petites volailles sur de simples ama ne sont vécues que comme des actes d'hommage ou d'offrande à une puissance singulière de la nature. Elles ne sont pas impérativement exigées, sous peine d'importants malhcurs, comme le sont de véritables sacrifices dus à une puissance surnaturelle en compensation de son travail.
} 
b) Deuxièmement, les ingrédients dont se composent les fétiches ne peuvent servir à les identifier. Ayant pris acte de l'importance fondamentale attribuée aux ama qu'ils contiennent (pas d'ama, pas de fétiche), il me sembla un moment souhaitable de les classer non plus d'après leur aspect, leur fonction ou le genre de cérémonies qui s'y rapportent, mais d'après leur nature même, sur la base d'une analyse de leur composition matérielle. Cependant il me fallut bien vite y renoncer pour les raisons suivantes:

- A quelques éléments différenciateurs près, ne dépendant souvent que de la flore locale, de nombreux fétiches d'une même classe, bien différents les uns des autres, se trouvent fabriqués en puisant dans un nombre restreint de plantes réputées pour leur puissance.

- Des fétiches de même nom, remplissant des fonctions identiques, ne comprennent parfois aucune plante commune.

- Les mêmes plantes peuvent servir à composer des fétiches renvoyant à des puissances appelées les unes $b o$, les autres vodu, n'ayant pas du tout le même statut. On explique alors que le bo travaille avec la même énergie ou la même force ( $\eta u s \hat{e}^{19}$ ) que le vodu.

- Il est toujours possible de suppléer au manque de force d'un fétiche en y incorporant une ou plusieurs nouvelles plantes choisies après avoir recueilli, à ce sujet, l'avis d'experts parfois très éloignés.

- Rien ne s'oppose, sur la base d'une connaissance approfondie des vertus des plantes, à une modification de la force de travail d'un fétiche.

En définitive les ama constitutifs d'un objet de culte ne caractérisent rien de plus que le moyen par lequel des hommes sont mis en rapport avec une puissance surnaturelle consentant à se laisser manipuler par eux. Ils représentent un type de force devant être employée pour modifier l'évolution naturelle d'une partie du monde mais n'identifient nullement le poste de commande d'une telle modification. Ce par quoi la puissance visée est elle-même désignée, ce sont les paroles qu'il est nécessaire de prononcer pour la mettre en éveil ou faire valoir son droit de l'employer. Il n'empêche que de telles paroles peuvent

${ }^{10}$ Le $\eta u s \hat{e}$ correspond, semble-t-il, à la notion yorouba d'ashè. 
encore être modifiées, allongées, abrégées, ou même traduites dans une autre langue, tout comme le nom décerné à une personne vivante peut être changé à condition qu'elle le veuille bien.

c) Enfin, contrairement à ce que certains ont prétendu ou continuent d'imaginer, la puissance d'un fétiche est jugée préexister et subsister quelque part, indépendamment de l'objet qui permet d'y accéder.

- Alors qu'en détruisant des ama, on supprime du même coup la puissance (d'attraction ou de répulsion d'influence) qu'ils développent, en détruisant ce qui matérialise un fétiche on ne supprime nullement la puissance (d'action) qu'il permet d'exercer. Rien n'interdit d'ailleurs, après profanation ou accident, de reconstituer au même endroit le même fétiche. Ce qui est essentiel dans sa transmission à un récipiendaire est l'introduction de celui-ci auprès de la puissance qu'il désigne, d'où résulte une aptitude à y faire appel avec succès. Il n'est pas rigoureusement nécessaire qu'un objet soit confectionné et lui soit alors remis si des instructions suffisantes sur la manière de se le confectionner lui-même peuvent lui être communiquées.

Au cas où la puissance a "saisi" directement l'intéressé, c'est-àdire a pris elle-même l'initiative d'établir le contact avec lui, ou si ce contact a déjà été établi par un ancêtre ou un esprit de brousse, aucune transmission initiatique par un être en chair et en os n'est indispensable. Même non initiée, toute personne disposant des connaissances adéquates pour avoir par exemple assisté un initié dans ses activités magico-religieuses, est habilitée à lui confectionner l'objet dont il aura besoin. Il arrive que le fils ou le frère d'un chef de culte (huns), qui a appris auprès de celui-ci les techniques nécessaires à l'entretien et au maniement de son vodu, installe lui-même ce vodu à l'une de ses épouses ou à l'un de ses enfants sur qui ce vodu s'est spontanément porté.

- Inversement, sans supprimer l'objet, il est possible de le neutraliser, lui et tous les exemplaires analogues qui en ont été diffusés, par suppression de la puissance à laquelle il se rapporte ou annulation de tout droit de l'utiliser. Des familles entières de bo ou de vodu dont l'usage s'est révélé nuisible à une communauté peuvent de la sorte être 
anéanties. Il suffit pour cela d'apporter l'un des objets qui les matérialisent ou leur sont intimement liés dans la forêt sacrée de la divinité royale Nyigblin, à Bè, ou bien chez l'un des prêtres ordinaires du même Nyigblin, à Togoville, où le responsable du culte exécute les rites nécessaires à son invalidation puis l'enterre sur place sous la bonne garde de Nyigblin ${ }^{11}$.

Non seulement le bo ou le vodu particulier ainsi enterré perd sa puissance, mais aussi, du même coup, tous les bo ou les vodu semblables dérivant de la même souche, c'est-à-dire appartenant à une même lignée ou une même arborescence de bo ou de vodu provenant d'un bo ou d'un vodu mère ayant été découvert et installé par quelque fondateur.

Ainsi, en 1951, le vodu Dadukodzo ("Kodzo, le serpent qui mord"), jugé trop méchant, fut acheté par Mr. Dogbonou, alors responsable de la forêt sacrée de Bè en l'absence du prêtre-roi, qui l'introduisit dans cette forêt pour y demander à Nyigblin son élimination. Tous les Dadukodzo qui avaient été installés dans la région perdirent aussitôt leur efficacité et furent peu à peu abandonnés.

La même année, les adeptes du vodu Atigeli (ou Tigari) eurent l'outrecuidance de se rendre à l'une de leurs cérémonies en traversant le quartier de Bè, où Nyigblin interdisait de jouer du tambour, tout en chantant et en battant leur tambour. Ils furent arrêtés en chemin et leur instrument, ainsi que le symbole du vodu qu'ils transportaient avec eux, furent confisqués. Une cérémonie secrète fut faite sur ces objets, à l'intérieur de la forêt sacrée, qui fit perdre aux Atigeli introduits dans la région toute leur puissance et découragea de continuer à leur rendre un culte.

Le même sort arriva en 1962 à une variété de bo de la catégorie des gabara. Un exemplaire de ce bo fut acheté par Mr. Dogbe

11 Un enterrement sans protection annule l'efficace du fétiche par dilution dans la masse terrestre. S'il arrive à quelqu'un, en remuant la terre, de découvrir par la suite les restes de ce fétiche, il ne trouve là que des choses sans importance. Cependant, s'il lui arrive de trouver pareillement les restes d'un Yèvhe-vodu ancestral (impossible à invalider par le même procédé), les âmes des ancêtres pourront le poursuivre jusqu'à l'obliger à reconstituer, c'est-à-dire à installer chez lui, l'efficace de ce vodu. 
Agbewonou, alors successeur de son cousin comme responsable de la forêt sacrée, qui l'enterra dans cette forêt après avoir excité contre lui la colère justicière de Nyigblin. Depuis ce jour, nul n'a plus entendu parler des méfaits de ce gabara dans la région.

Que le fétichisme ne puisse être réduit ni à un culte des objets ni à un culte des propriétés cachées des objets, mais vise une réalité essentielle dont l'objet permet seulement de s'approcher, devient évident en remarquant qu'il serait totalement inutile d'y recourir si l'on était assuré d'obtenir les mêmes bienfaits avec de simples ama.

Ceux-ci permettent de filtrer, concentrer ou détourner certains flux d'acheminement sur terre des phénomènes, de profiter astucieusement de leur énergie au lieu de leur résister vainement, de remédier à certaines imprégnations malsaines attirant sur soi de la malchance, etc., bref de naviguer au mieux de ses intérêts parmi une multitude d'influences.

Comment assumer encore mieux la direction de sa destinée si ce n'est en maîtrisant de tels flux à leur source même, en ne se bornant plus donc à composer avec ceux qui sont en train d'arriver, mais en acquérant le droit de décider de ceux qui vont être envoyés. Après n'avoir tenté, dans un premier temps, que d'infléchir en sa faveur, dans les limites du possible, l'évolution des événements, on est amené, tôt ou tard, à vouloir modifier aussi la nature et l'étendue du possible. L'opération se traduit par l'assignation de nouveaux objectifs aux agents spirituels par l'activité desquels on est ou on risque d'être affecté $^{12}$. Elle suppose que l'on soit mis en état de faire appel à des puissances extérieures à celles qui assurent le fonctionnement automa-

12 Il est généralement impossible de savoir si tel ou tel résultat est dû au détournement d'un événement (grâce à des $a m a$ ) vers une possibilité peu probable, ou à sa précipitation vers une nouvelle possibilité très probable ayant été suscitée par une divinité fétiche ou par une divinité ancestrale. C'est à un devin qu'il revient d'en avertir les intéressés. 
tique du monde terrestre, méritant par conséquent d'être qualifiées de surnaturelles $^{13}$.

De telles puissances sont de deux sortes:

- Celles touchées au moyen d'objets de la catégorie des vodu décident de l'émission des ordres et des souffles créateurs qui déterminent la progression des choses. Elles modifient les impulsions qui poussent les êtres à se dépenser. Il s'agit de puissances célestes intelligentes se partageant à tour de rôle, conformément aux révolutions du soleil, de la lune et des étoiles, le contrôle du débouché des entrailles de la terre.

- Celles touchées au moyen d'objets de la catégorie des bo ne sont pas comme les précédentes incitatrices de certains types d'événements mais vont aviver, subjuguer ou ligoter les esprits (des vivants ou des mauvais morts) qui interviennent passionnément dans les affaires du monde. Au lieu de modifier les impulsions qui parviennent à des esprits, elles modifient directement et sans délai leur réceptivité. Elles paraissent consister en réserves de dispositions intimes à s'engager ou non dans tel ou tel genre d'activité ou à éprouver telle ou telle sorte d'expérience.

\section{Dans quelle perspective sont-ils employés ?}

Essayons de comprendre à présent comment des ingrédients matériels permettent de trouver accès auprès de puissances se laissant

${ }^{13}$ Me paraît qualifiable de sumaturel tout ce qui relève de la capacité d'un sujet d'agir librement, en se jouant des déterminations physiques, psychiques, sociales, historiques, culturelles, etc. Nul n'est réellement libre, en effet, que dans la mesure où ses comportements se trouvent raccordés à un ensemble de causes, séparées de leurs effets, inaffectées par ceux-ci. Quclle qu'en soit la localisation symbolique au ciel, une telle transcendance est dans l'homme. Elle coïncide avec son droit de conquérir une position surélevée vis-à-vis de la nature, depuis laquelle il peut en diriger intelligemment l'évolution. De puissances surnaturelles, un individu peut recevoir des inspirations ou des conseils (notamment des indications concernant l'emploi d'ama), mais aussi des aides concrètes ou spirituelles (par les bo et les vodu). 
actionner par des formules de nomination, et surtout quel est l'aboutissement ultime de leur emploi ${ }^{14}$.

Nous avons vu que l'existence de l'homme était prédéterminée par les paramètres d'une origine assez arbitraire. Il est jugé avoir été envoyé au monde pour réaliser un projet d'exploitation d'un invisible "champ" ensemencé de germes d'événements et d'expériences. Ce que définit un tel projet constitue l'objet fondamental de ses désirs. Une fois séparé de lui, après rupture du cordon ombilical, il ne peut plus le remanier. Il se retrouve placé à son service et ne peut plus que se soumettre aux sortes de directives maternelles qui en émanent. Il lui reste néanmoins la responsabilité d'adopter l'une des deux attitudes suivantes:

- soit une station animale à l'horizontale, dans le sens du flux naturel qui pousse, d'est en ouest, les événements à se produire. Mais cela lui laisse encore le choix entre une condition enfantine ou sauvage d'être non réfléchi, subordonné aux contraintes matérielles et sociales comme à ses propres inclinations, et une condition adulte ou civilisée dans laquelle il cherche à remédier à son sort, exécute pour cela des sacrifices ${ }^{15}$, mais s'abandonne totalement sur ce point à l'autorité de ses ancêtres.

- soit un redressement humain à la verticale, dans une condition supérieure de sage lui permettant d'assumer lui-même, en prenant l'initiative de sacrifices, une autorité qui lui revient de plein droit sur la conduite de sa vie.

a) Il peut fort bien en effet se contenter d'obéir automatiquement aux impulsions qui, depuis l'orient, lui parviennent des profondeurs et paraît alors subir passivement sa destinée. En réalité, il demeure

14 Il ne sera pas question, ci-dessous, des buts poursuivis et des résultats obtenus par les clients du magicien féticheur. Nous n'envisagerons que les fruits, bien différents, des pratiques de ce dernier sur sa propre personne. Après avoir souvent surmonté une épreuve, il a acquis, par delà une position passive de demandeur de secours, une position active de détenteur d'une puissance maîtresse des situations correspondantes.

${ }^{15}$ Du point de vue évhé, comme de bien d'autres populations d'Afrique noire, agir équivaut à sacrifier. On ne saurait en effet remanier le fonctionnement normal du monde, de la société et des hommes sans établir de rapports avec des puissances surnaturelles et sans se charger, en conséquence, d'obligations sacrificielles. 
l'artisan obstiné de ce qui lui arrive. Une véritable passivité se traduirait par un abandon de poste de travail, c'est-à-dire une profonde dépression ou la mort. Or nous le voyons subir sans révolte la loi du monde et se comporter en esclave zélé du Créateur. La puissance de la nature ne le pousse à agir que dans la mesure où il y consent. Si l'énergie par laquelle il est poussé de l'avant n'était pas soutenue par une irrépressible volonté de se dévouer à quelque chose, il cesserait de contribuer à la production du moindre événement.

C'est en définitive une force qui le lie à des conceptions demeurant secrètement emmagasinées dans le "monde de l'origine" qui est responsable de la situation dans laquelle il se trouve. Car rien ne le pousserait s'il n'adhérait pas à la source des poussées qui relancent continuellement son activité. Rien ne saurait le préoccuper à défaut d'une impulsion constitutionnelle à exister, l'engageant à répondre par une telle adhésion à la séduction exercée sur lui par une réal ité complémentaire de la sienne. Il se sent provoqué par cette dernière comme peut l'être un mâle par une femelle, un cultivateur par la terre de ses champs, ou un artisan par des matériaux à travailler. Sans son empressement à aller interférer, au risque de s'y perdre, avec une nature radicalement autre que lui-même, rien ne se passerait.

Au fondement de l'existence conjointe des agents spirituels et du monde terrestre au parachèvement duquel ils se dévouent réside donc un principe de mise en relation féconde de l'En-haut (le Ciel, Dzi, la patrie de ces agents) et de l'En-bas (la Terre, Anyi, servant simultanément d'entrepôt de leurs conceptions et de lieu de réalisation progressive de celles-ci) au sein d'un champ de force où l'un et l'autre se répondent. Les esprits réagissent par application de leur attention et par des actions aux attraits de la Terre. Celle-ci, à son tour, réagit à leurs actions par une modification du pouvoir de séduction de son apparence. C'est ce désir mutuel d'union, cette exigence d'interaction équilibrée, qui est responsable de l'unité de l'univers par application l'une sur l'autre des deux moitiés de la grande calebasse qui le représente.

A la poussée génésique de la Terre, à la puissance d'avènement de ses productions, à ce qui s'y apparente à l'antique notion d'un pneuma matériel, répond en écho un désir d'emprise des esprits sur 
elle et vient se mêler une effusion directrice n'aboutissant nullement à un écrasement autoritaire de ses propres facultés mais à la mise en chantier d'une auvre commune.

Le "souffle" vital, atmosphérique, qui véhicule toute chose à la surface de la terre et l'y maintient en état symbolise donc au sens fort un "souffle" spirituel, éthéré, qui précipite les esprits dans le sein de la génitrice universelle par avidité pour l'existence. L'un et l'autre représentent les deux sections artificiellement brisées du trait d'union toujours reconstituable entre l'En-haut et l'En-bas ${ }^{16}$.

Telle est la donnée primordiale, antérieure à toute conception et toute imagerie mentale, qui explique que l'efficacité des symboles sacrés est bien supérieure à celle des signes, des images ou des allégories. A chacun de la mettre à profit pour arriver à toucher et à maîtriser, comme au moyen d'un outil, certaines des puissances par lesquelles il se ressent dominé.

En s'imprégnant volontairement d'un certain aspect du souffle terrestre, défini par des fragments de corps et d'objets, et en l'attisant en soi-même par certains rites, il devient en effet possible d'entrer en rapport vivant avec l'aspect correspondant du souffle spirituel et, à travers lui, de s'élever jusqu'à sa source, au niveau des puissances subordonnées à l'Esprit universel (Mawu) qui ont reçu pour mission de régenter ce qui se passe sur terre. Telle est la fonction des objets fétiches. Ils servent de base à un travail d'accession au monde surnaturel.

b) L'existence de l'homme dominé par ses pulsions, esclave de son moi, est déjà une production sensée, signifiante de quelque chose puisque se rapportant à des référents élaborés par lui dans le monde de l'origine. Cependant elle ne s'apparente qu'au discours d'un rustre et

16 Ainsi est résolue dès le départ, dans la pensée des Evhé, l'opposition entre un pneuma matériel, vital ou psychique, du genre de celui postulé par Plutarque dans la production des oracles de la Pythie, et un pneuma spirituel d'origine transcendante, animant notamment les prophètes, telle qu'elle est notamment avalisée par Gérard Verbeke (1945) à l'appui d'un préjugé favorable aux conceptions judéo-chrétiennes. Leur notion de respiration ( $g b$ ogbo, ce qui va et vient) caractérise essentiellement l'unité d'un mouvement d'alternance et d'échange entre l'énergie matérielle ou terrestre et la force spirituelle ou céleste. 
non à celui d'un homme cultivé. L'ambition peut donc lui être inspirée de ne plus se contenter de laisser les choses se faire toutes seules, mais d'exploiter au mieux les possibilités qu'elles adviennent.

Conscient de sa faiblesse, il peut s'en remettre à des médiateurs défunts qui, ayant regagné le monde de l'origine, sont capables d'y voir clair et d'y opérer en son nom divers remaniements. Cependant le recours à de tels médiateurs, dont le rôle majeur est de préserver la continuité des traditions, présente le désavantage en même temps que l'avantage de faire prévaloir les intérêts de la collectivité sur ceux des individus. Quiconque s'en remet à eux n'est certes plus uniquement soumis aux exigences de son moi, mais devient agi par les forces constitutives de son groupe, et cela ne vaudrait guère mieux si les operrateurs en question ne devaient pas eux-mêmes aller puiser leur force et leurs raisons d'agir auprès de puissances transcendantes.

c) L'idéal est que, déjà éventuellement poussé de l'avant sur la bonne voie par les défunts sous la direction desquels il s'est placé, le sujet parvienne à se gouverner intelligemment lui-même en s'adressant à des puissances du même ordre que celles des divinités contrôlées par ses ancêtres. Or voilà ce à quoi il lui est immédiatement proposé de s'entraîner au moyen de fétiches qui lui permettent notamment de cultiver à sa guise son champ de ressources originelles ${ }^{17}$.

En profitant des correspondances symboliques entre les souffles d'en-bas et les souffles d'en-haut, entre une énergie matérielle subtile et une force spirituelle qui se présentent comme les deux faces d'une même tension vitale, il lui est permis de remonter artificiellement jusqu'au niveau où siègent les puissances célestes. Tel est cependant son aveuglement intellectuel qu'il a besoin d'être mis en rapport avec elles, sa main dans la leur, par quelqu'un d'autre qui lui enseigne la façon de les actionner.

De telles puissances supérieures ne présentent d'intérêt pour l'homme que si elles ont toute liberté de modifier à son avantage

${ }^{17}$ On remarquera qu'une telle culture du discours par lequel un homme manifeste automatiquement les ressources d'un "moi" érigé en objet fondamental de ses désirs se pratique au moyen d'actes religieux. Elle ne doit pas être confondue avec un entraînement intellectuel ne menant qu'à assimiler un certain genre de rationalité. 
l'évolution des événements qui le concernent. Or il leur faut mobiliser à cet effet l'énergie du champ d'interaction qui les maintient en liaison avec les mondes terrestres en même temps qu'il les en écarte. Dans ces conditions, leur souveraineté ne saurait être effective que si leurs décisions ne sont en rien déterminées par le contenu d'un tel champ. Il apparaît donc qu'elles le transcendent également. C'est pourquoi un simple savoir magique ne donne aucune prise sur elles. Alors qu'un simple utilisateur d'ama tire quasi-scientifiquement profit des vertus cachées, mais bien réelles, des choses, un féticheur doit remonter a travers les forces qu'elles symbolisent jusqu'aux agents capables de modifier la façon dont le système constitué par le monde de l'origine (amedzophe), le monde de la vie sur terre (kodzogbe) et le monde atmosphérique intermédiaire entre eux deux ${ }^{18}$, animé et rendu sensé par des agents spirituels (luvho), arrive à fonctionner tel qu'il est livré à lui-même. Alors qu'il suffit de se faire enseigner comment utiliser des ama, on a besoin d'être initié à tout bo ou à tout vodu que l'on se procure.

L'objet de culte correspondant ne suffit donc pas à capter des bienfaits divins. Il n'est attracteur que d'une force permettant d'accéder à une source de tels bienfaits. Il ne constitue pas l'organe d'action d'une puissance, mais un moyen d'entrer en relation directe avec elle. Une fois contrainte à l'action par des paroles adéquates, celle-ci réunit elle-même les moyens de provoquer, souvent ultérieurement et à distance, ce qu'un officiant investi du droit de s'en servir lui commande de faire.

${ }^{18} \mathrm{C}$ 'est aux flux atmosphériques que viennent se mêler les influx spirituels provenant du ciel, de sorte que l'atmosphère, lieu de communication entre le monde de l'origine et le monde de la vie sur terre, sert aussi d'intermédiaire entre les mondes d'en-bas et le monde d'en-haut. On l'appelle en évhé xéxéme, c'est-à-dire "l'intérieur (me) du barrage ou de la fermeture" (xé signifiant barrer, fermer, clore) des réalités terrestres sur elles-mêmes. Cependant, si elle délimite ainsi ces réalités, elle n'en est pas moins jugée les pénétrer entièrement. Pour contrôler ce qui se passe ici-bas et éventuellement y modifier quelque chose, c'est en elle que s'insinuent les vodu (ou plutôt la force qu'ils emploient) avant de revenir périodiquement rendre des comptes au maître divin (c'est-à-dire en repliant leurs forces auprès de Mawu qu'en réalité ils ne quittent pas). 
Tout bien considéré, l'homme qui s'adonne à la magie fétichiste apprend à recouvrer le statut dont il bénéficiait avant de prendre corps dans le sein de sa mère. On dit couramment qu'il y a été conçu. En réalité c'est lui qui y a conçu l'objet même de son existence, objet auquel il se dévoue et s'identifie par la suite au point de le considérer comme un "moi" auquel tout ce qu'il éprouve et réalise est finalement rapporté. Cette identification (erronée) de lui-même à un tel objet l'amène à méconnaître, à défaut de recul, que ce qui se répète sous diverses formes dans son existence n'est qu'une chose qu'il aurait tort de renier mais est invité à travailler religieusement. Elle le fait se présenter au monde comme l'enfant d'une mere, alors qu'il demeure essentiellement un époux de la Mère. De géniteur qu'il était, il se retrouve en position d'engendré. Son initiation religieuse aura notamment pour but de lui révéler qu'il n'existe qu'en relation avec la génitrice universelle ${ }^{19}$ dont, paradoxalement, il est simultanément, dans des proportions alternantes, l'amant et le fils.

On pourrait craindre que l'impulsion initiale qui le porte à s'unir au partenaire qui lui fait face n'aboutisse à la catastrophe d'une fusion narcissique avec une sorte d'image de lui-même gravée dans la substance génératrice. Fort heureusement son premier acte d'union à la Mère n'aboutit qu'à la définition en son sein d'un projet prénatal qui ne lui servira, tout compte fait, que de prétexte à entrer dans l'existence en y jouant un rôle quelconque. C'est que le principe d'unification n'est pas un principe de fusion mais un principe de relation féconde entre deux termes conservant non seulement leur identité mais s'enrichissant mutuellement de leurs rapports. Au lieu que le mouvement ne vienne s'arrêter sur la cible visée, il va indéfiniment se poursuivre en étant centré sur elle. D'une position initiale où le sujet était situé au-dessus (quand, venant du Ciel, il concevait son projet), il va passer au-dessous (en devenant sur terre serviteur de ce projet). Et d'une position où il se retrouve au-dessous (au cours de sa vie sur terre), il va tendre à repasser au-dessus (immédiatement, par magie, ou

${ }^{19}$ On la nomme le plus souvent, en évhé, Bomeno, ce qui signifie "la mère $(n o)$ du lieu (me) du champ (bo) de l'au-delà". 
dans l'attente d'une élévation finale au Ciel $)^{20}$. Cette invitation à un va-et-vient, analogue à celui de la respiration ( $g b \ni g b s$ ), qui unit dynamiquement l'En-haut et l'En-bas est le principe de toute évolution spirituelle.

Une fois né, une fois acheminé sur terre en condition animale par un flux horizontal parcourant d'est en ouest la surface terrestre, le voici engagé à regagner sa condition céleste, paternelle, d'émanation de l'Agent universel, et à se redresser pour cela à la verticale. La réalisation du grand mystère de l'existence va consister pour lui à assumer simultanément sa condition de fils et de père centrée sur une réalité maternelle à la fois cause de sa "chute" (son implantation dans le monde d'en-bas) et provocatrice de son salut (le recouvrement de sa souveraineté $)^{21}$. Il ne pourra cependant y parvenir qu'en acceptant le secours d'un alter ego déjà initié, d'un maître spirituel ou d'un ancêtre, agissant eux-mêmes au nom de l'aspect du Dieu suprême appelé Mawugã Segbo ("le grand Dieu, boule des $s e ")$ qui est chargé de déléguer auprès de chaque homme un génie (appelé se) l'aidant à accomplir a mieux sa destinée.

En actionnant des puissances paternelles et en le faisant normalement sous couvert d'un génie bienveillant (notamment l'un des se-kpoli intervenant en divination par Afa) ou d'un vodu (Agè ou toute autre puissance qui en dérive) spécialisé dans la production d'oracles et la fourniture de secours aux magiciens professionnels, le sujet se les assimile peu à peu et les changements d'état de conscience qui en résultent peuvent nous être présentés comme une remontée de l'échelle

20 Cette élévation n'intervient normalement qu'à l'issue d'une longue période pendant laquelle un défunt exerce des fonctions d'ancêtre ou est associé à leur exercice. Elle est cependant immédiate pour les rois défunts (qui ne sont pas ancestralisés) et pour certains hommes d'exception qui avaient acquis de grands pouvoirs magiques (ils disparaissent du monde sans laisser de trace, comme le firent Gbaku et Dutokonyi à Anloga, ou s'embarquent eux-mêmes pour l'au-delà, comme le fit Tsali, à Tsiame, sur le dos d'un crocodile).

${ }^{21}$ Notons que, même à un âge très avancé, un féticheur se reconnaît lui-même, et cela précisément au moment où il entre en rapport avec le sumaturel, dans une condition analogue à celle d'un petit enfant totalement livré à la merci de sa mère. 
mystique chromatique qui conduit une âme de l'obscurité intellectuelle du monde terrestre à l'éclatante blancheur du sommet des cieux ${ }^{22}$.

Grâce à des objets en lesquels signifiés et signifiants, référents intelligibles et réalités concrètes ou imaginaires, ne se télescopent à l'horizontale que pour mieux renvoyer à la verticale, un féticheur se présente idéalement, en définitive, comme un surhomme osant braver symboliquement l'interdit de l'inceste, revendiquant une possession sans intermédiaire du corps de sa (la) Mère, ne cherchant en aucun cas à régresser à l'intérieur de son sein mais à y rentrer à la manière d'un amant, avec seulement son pénis, en conservant vis-à-vis d'Elle une position dominante. En nous affirmant que "le prototype normal du fétiche est le pénis de l'homme", Freud ${ }^{23}$ paraît avoir eu l'intuition de ce qui est ainsi fondamentalement en jeu dans la relation individuelle à un objet de culte. Son rôle n'étant pas d'assister des mystiques mais de guérir des patients, il n'en a cependant retenu que ce qui s'en laissait entrevoir sur le plan de la relation du vivant avec le terrain d'enracinement de ses désirs. Au lieu de concevoir le fétiche comme un moyen de recouvrer une virilité primordiale, il en a fait un substitut du pénis imaginaire de la mère, c'est-à-dire un moyen de dénier l'absence de pénis chez celle-ci. Selon pareille acception, ce qui aurait dû être élaboré en instrument de délivrance ne l'est plus que comme palliatif à l'angoisse de castration résultant d'une tentative de maintien d'une relation fusionnelle du sujet avec sa génitrice.

\section{Quels en sont les équivalents éventuels ?}

Les fétiches, qui ne sont ni de simples autels, ni de simples émetteurs ou redistributeurs d'influences, peuvent finalement être définis comme des objets de culte qui, grâce aux vertus subtiles des ingré-

${ }^{22}$ Au banangolo bambara, symbole de la remontée serpentine jusqu'au sommet du ciel en traversant sept zones de couleur (cf. Youssouf Cissé, 1973: 139-142, 172 et 176-177), correspond, à l'ancienne Côte des Esclaves, le vodu "Serpent arc-en-ciel".

${ }^{23}$ Gesammelte Werke, p. 317 ; repris dans "Objets du fétichisme", Nouvelle Revue de Psychanatyse, $\mathrm{n}^{\circ} 2,1970$, p. 24. 
dients dont ils se composent, permettent à ceux qui en ont pris charge d'accéder directement, sans dépendre donc des caprices ou du bonvouloir d'esprits de la nature ou d'âmes désincarnées, à des sources d'action surnaturelles.

Le pouvoir de leurs ingrédients - identiques à ceux des médicaments magiques, gris-gris, amulettes ou talismans - provient du fait qu'ils restent marqués par le type de force avec lequel un esprit s'est engagé dans le monde pour y produire des signifiants dont ils sont des traces ou des extraits. Pareille force n'est pas directement décalquée dans leur substance ou leur structure perceptible ou mesurable, apparemment inerte, mais dans un insaisissable "souffle" vital qui leur est inherent $^{24}$. C'est en lui seulement, ou par son opération, que la terre peut être considérée comme l'image inversée ou en négatif du ciel et que, dans le spectacle des choses, miroitent des puissances transcendantes.

Du fait que la vie apparaît particulièrement proliférante dans le règne végétal et que son développement apparaît conditionné par les productions végétales, il n'est pas étonnant que, chez des populations en étroit contact avec la nature, ce soient des ingrédients végétaux qui aient été jugés les plus aptes à établir une jonction avec des puissances correctrices ou directrices du devenir du monde. Néanmoins rien ne s'oppose à une utilisation dans le même but de n'importe quel fragment d'objet signifiant ou de corps, pourvu qu'il ait reçu l'agrément de la puissance visée. D’éventuels remplacements des herbes ou des feuilles

${ }^{24}$ Du fait que toute croissance de corps et toute production de phénomène est jugée dirigée par un esprit conformément aux ordres créateurs qui lui parviennent depuis la "bouche" de la Terre, le fétichisme du sud-Togo apparaît fondé sur l'animisme. Comme son efficacité est tributaire d'un principe universel de mise en relation des contraires, réalisateur de l'unité du monde, il apparaît aussi fondamentalement monothéiste. Comme il vise à activer une pluralité de puissances surnaturelles trônant symboliquement au ciel, il est également, à sa manière, polythéiste. Dans la mesure où il estime que le suprême principe unifiant est cause d'un souffle nourricier submergeant fluidiquement toute chose et tout être obligé de le respirer, il est en outre panthéiste. En admettant un attachement persistant des esprits mal désincarnés (car prématurément arrachés sur terre à leur poste de travail) aux moyens spirituels qu'ils avaient réunis pour exister, il apparaît enfin polydémoniste. Bref il intègre en lui toutes les étapes pouvant être distinguées par une théorie évolutionniste des religions. 
par des choses ou des prélèvements d'un autre genre ne sont cependant possibles que conformément à certaines valences symboliques et à une certaine logique. Il importe en particulier que la population ait été fortement concernée par les nouveautés prises en considération ${ }^{25}$.

Quantité de restes d'objets à usage spécifique (navette de tisserand, morceau de vieux moteur, etc.) ou de traces d'événements dans lesquels les hommes ont été impliqués (tickets de train, feuilles tombées d'un arbre sous lequel ont été jugées des affaires d'assassinat, etc.) sont effectivement récupérés à des fins magico-religieuses. Ils témoignent d'une disposition à rechercher certaines experriences ou d'une atmosphère favorable à un certain type d'activité. Toutefois ils se prêtent mieux à évoquer l'imposition à un esprit d'une intention qui lui est étrangère et conviennent donc surtout à la confection des bo, destinés à affecter les esprits impulsés, plutôt qu'à celle des vodu, employés pour modifier en amont les impulsions qui leur parviennent.

Nous observons par ailleurs que les ingrédients naturels évocateurs d'un certain type de vitalité sont aujourd'hui complétés ou remplacés sans aucun problème par divers symboles des capacités, de la richesse, des connaissances et du haut statut social des commerçants musulmans (cas des goro-vodu d'origine prétendument haoussa, consommateurs de grosse cola) et des Blancs ayant jadis abordé le pays par la mer (cas des cultes de Mami Watta).

Pour nous mettre en état de traiter du problème des fétiches dans toute sa généralité, étendons-nous cependant plutôt sur des équivalents susceptibles d'être utilisés ailleurs, jusque dans d'autres continents, pour confectionner des objets de culte analogues offrant parfois une tout autre apparence. De toute évidence il pourra s'agir:

- De morceaux de texte, de préférence écrits à la main dans l'intention requise. Ils sont d'autant plus efficaces qu'ils ont été composés ou recopiés par des sujets spirituellement puissants. Les plus efficaces entre tous sont évidemment des morceaux de textes sacrés, notamment des passages du Coran supposés avoir été énoncés par le

${ }^{25}$ Ce qu'a notamment souligné Taoufik Adohane (1991: 55-73). 
prophète sous la dictée divine ${ }^{26}$. Nous en trouvons inclus dans les objets sacrés du culte des goro-vodu.

- De tracés auxquels une tradition savante prête une signification adéquate. En particulier ceux qui évoquent des messagers spirituels de caractère défini ou des types d'influx spirituels comme ceux ayant été attribués aux astres. Ils se révèlent d'autant plus puissants qu'ils ont été gravés, conformément à un système de correspondances symboliques, à des moments et sur des matériaux ayant de l'affinité avec ce qu'ils représentent.

- D'arrangements ou de configurations de lettres n'ayant aucune vocation à désigner quoi que ce soit mais constituant, selon une certaine conception de l'origine et de la structure de l'alphabet, de véritables formules d'influx spirituels particuliers. Alors que le souffle est considéré comme le véhicule matériel subtil de la parole, les lettres et les phonèmes qu'elles composent peuvent représenter en effet son encadrement par des forces spirituelles.

- En dépit d'une fixation indésirable de l'esprit sur les formes intelligibles correspondantes, rien ne s'oppose à ce que soient utilisés, non plus de simples fragments de corps, mais des cadavres entiers de petits animaux réputés pour leur vitalité, leur courage ou leurs capacités remarquables ${ }^{27}$. Ils garnissent abondamment les étalages des "marchés des fétiches" de Lomé et de Vogan.

- Rien ne s'oppose non plus à l'utilisation de sculptures et de dessins propres à évoquer les vertus attribuées à de tels animaux, à certains types d'homme, certains personnages historiques ou certains modèles divins. Néanmoins il demeure préférable, en la matière, d'utiliser plutôt des schémas ou des diagrammes ne conservant de la

26 "La récitation coranique, notait pertinemment Constant Hamès, s'inscrit ici (pour une certaine pratique magique) dans une démarche très particulière de médiations instrumentales à effets contraignants qui relève d'une sorte de fétichisme de l'écrit et de la parole" (1987: 317)

${ }^{27}$ Cela toujours, bien entendu, sans la moindre intention d'asservir et d'utiliser leurs esprits (luvhs) désincarnés. Dès lors qu'une créature animale (parfois jadis humaine) est enterrée vivante, ou aussitôt après avoir été mise à mort, sous un vodu, c'est pour que son esprit puisse être mis au travail par la puissance indépendante du fétiche. 
réalité correspondante, réelle ou archétypique, que la force de sa structure.

Nous étions partis de corps et d'objets. Nous voici arrivés à des figurations et à des graphismes. Or il ne semble pas interdit d'aller encore plus loin en admettant comme signifiants utilisables de pures représentations mentales. Il suffira que, pour les besoins du culte, elles puissent être rapportées à quelque chose.

Je m'empresse d'avouer que les Evhé n'ont développé aucune pratique prenant ainsi appui sur des formes imaginées ${ }^{28}$. Ils admettent pourtant que le monde où nous vivons comprend aussi bien des réalités mentales (uniquement produites par l'esprit de l'homme qualifié d'esprit de mort, $k u$-luvhs, du fait qu'il subsiste sur terre après la mort) que des réalités tangibles (produites par tous les esprits non humains et par l'esprit vital ou agbe-luvho de l'homme). Pour qui se situe à leur point de vue, il est évident que des images mentales non fantaisistes, élaborées conformément à des normes culturelles et se rapportant de ce fait à quelque chose subsistant quasi-objectivement en dehors du sujet, sont propres à remplir les mêmes fonctions que les ingrédients habituels des fétiches. Au lieu que l'opérateur ait à s'imprégner des vertus d'un objet matériel, il doit cette fois s'imprégner des vertus d'une pensée déterminée puissamment recréée en lui ${ }^{29}$. Comme tout le processus d'accession au surnaturel se déroule en définitive à l'intérieur du sujet, tenu d'entrer sacrificiellement en osmose vitale avec un objet de culte extérieur, le procédé paraît même susceptible d'être bien plus efficace. Il faut et il suffit pour cela que des disciplines d'entraînement a la concentration mentale, permettant de faire exister devant soi, de manière autonome, des formes signifiantes, aient fleuri dans le milieu culturel ambiant et soient sérieusement adoptées.

28 Il n'en reste pas moins que de telles pratiques sont en train de s'implanter aujourd'hui dans certains milieux instruits de Lomé.

${ }^{29}$ Notons, à ce propos, que les Adja-Evhé, comme leurs voisins yorouba, traitent la tête de l'homme, siège de la pensée symbolique, comme un autel de vodu, c'est-à-dire comme un objet singulièrement chargé de souffle. Ils sacrifient dessus et luioffrent à manger, notamment avant une initiation, pour la purifier et la fortifier. 
Tel est le cas en magie d'origine indienne, et plus spécialement tantrique, où des visualisations de divinités, de mandala, de diagrammes sacrés ou de centres subtils du corps (destinés à capter des influx spirituels $^{30}$ viennent avantageusement remplacer statues, peintures et graphismes, ou tiennent lieu du moins d'instrument magique essentiel $^{31}$.

A la limite, des énoncés oraux paraissent également susceptibles de remplacer des morceaux de texte. Des paroles prononcées à voix haute, selon les règles, possèdent en effet des propriétés objectives sur lesquelles il est possible de se fonder pour introduire et cultiver en soi un "souffle" correspondant. Celles à retenir devraient toutefois ne pas figurer parmi les discours et les expressions liturgiques accompagnant l'exécution du rituel $^{32}$. Mais comment les distinguer alors des paroles efficaces, plus ou moins mystérieuses (telles les gbesa ou les $\eta$ k.phopho des Evhé), destinés à nommer, éveiller et forcer à agir les puissances correspondantes avant de leur préciser ce qu'on attend

\footnotetext{
${ }^{30}$ Le corps de l'adepte lui-même, travaillé de façon à être parfaitement équilibré et pouvant alors être considéré comme un talisman parfait (image du modèle céleste de l'homme), peut être pris pour fondement d'une pratique spirituelle le rendant analogue à un fétiche. Ce qui tient lieu d'objet (une structure microcosmique fortement signifiante) est alors d'emblée intériorisé. Le sacrifice l'est aussi et se résume en une discipline ascétique. Même la parole d'activation des puissances surnaturelles correspondantes demeure intensément concentrée dans l'esprit. Elle y est réduite à un cri d'appel silencieux ou au murmure incessant de l'acte respiratoire (notion indienne d'ajapa-japa).

31 Apparemment la visualisation mentale indienne, fruit de la bhâvanâ, déposée sur un support (linga, mandala, yantra, objet commun, corps humain ou statue) est analogue aux ingrédients essentiels introduits dans l'enveloppe ou sous la figuration d'un bo ou d'un vodu. On pourra en juger en se référant aux études rassemblées par André Padoux (1990), notamment à celles d'Hélène Brunner et à la sienne.

32 Nous pouvons considérer de telles paroles liturgiques, ainsi que toutes les séquences des rites focalisés sur l'objet (gestes, énoncés, images induites), comme des éléments occasionnellement ajoutés à celui-ci, à situer sur le même plan que sa forme visible et ses accessoires décoratifs, uniquement destinés à ouvrir la voie vers la vertu de ses ingrédients essentiels.
} 
d'elles ${ }^{33}$ ? Serait-il possible que de telles paroles aient une double fonction?

Certaines formules sacrées de nomination des puissances possèdent effectivement une structure signifiante assez sophistiqué pour pouvoir à la fois symboliser un certain type de force spirituelle et identifier l'entité surnaturelle au service de laquelle cette force est placée ${ }^{34}$.

Mais qu'un même énoncé soit capable de nous renvoyer simultanément, ou successivement dans la même foulée, à plusieurs niveaux nous amène à nous demander si, inversement, certains matériaux, tout au moins des écrits, ne serviraient pas dans certains cas à nommer secrètement des puissances plutôt ou aussi bien qu'à symboliser les forces spirituelles qu'il leur faut employer pour passer à l'acte. Rien ne semble s'y opposer hormis une affinité plus prononcée des paroles avec les essences ou les quiddités, et des objets concrets avec les forces de réalisation ou d'expression. S'il est vrai que des puissances ne semblent pas correctement nommables au moyen d'objets naturels entassés les uns sur les autres, nous ne rencontrons pas moins certaines d'entre elles correctement désignées en leur essence même par des écrits numériques.

En Afrique noire, le long de la côte qui s'étend du Ghana au Nigéria, les signes géomantiques d'Afa identifient, sous forme de nombres binaires, les 256 variétés de génies célestes aidant chacun à accomplir au mieux sa destinée. De la poudre divinatoire sur laquelle

33 Toutes les prières adressées aux bo et aux vodu se subdivisent en (a) une première partie invariante, toujours cérémoniellement enseignée à un récipiendaire en même temps que lui sont communiqués les autres secrets relatifs à la confection et au soin de l'objet de culte, (b) un développement variable, composé en fonction des circonstances à l'initiative de l'officiant, rendant hommage à la puissance évoquée et lui exprimant le plus clairement possible, mais en usant souvent de formules consacrées, ce que l'on attend d'elle. Cette seconde partie de la prière définit une structure de captation et d'orientation du flux de force agissante déclenché par la première.

34 Tel est le cas des grands mantra indiens. Il arrive à un grand nombre d'entre eux d'être partagés en plusieurs sections dont chacune résume la possibilité pour une entité surnaturelle de subsister en son principe comme de se manifester plus ou moins loin hors d'elle-même. Leurs deux ou quatre parties (appelées bîja, pinda, samjinâ, pâda) les développent depuis leur "âme" jusqu'à leur "corps" en passant par une double phase intermédiaire (voir notamment à ce sujet Sanjukta Gupta, 1989: 235). 
ils ont êté tracés est souvent déversée sur le contenu d'offrandes abandonnées appelées vəsa et se trouve ajoutée aux ingrédients de certains fétiches.

Les 266 signes graphiques bambara leur correspondent si nous voulons bien y ajouter les dix éléments, figurés dans le vodu Gbadu, qui sont considérés comme leurs mères. Or ils entrent de leur côté dans la composition des objets de culte appelés boli qui font fonction de fétiches.

Dans le monde islamique, nous rencontrons assez systématiquement des configurations géométriques de nombres. Ceux-ci nous y sont présentés comme l'âme des lettres qui en sont le corps ${ }^{35}$. On connaît par ailleurs l'affinité entre l'essence et le nombre. Un nombre est une forme épurée à l'extrême, disposée à aller se communiquer aux productions les plus élémentaires de la nature pour nous les rendre prévisibles, calculables, intelligibles. Le contenu d'un énoncé, immédiatement supporté par du souffle et des lettres, nous apparaît ainsi enraciné dans des principes jouissant vis-à-vis de lui d'un recul analogue à celui des agents divins par rapport aux choses terrestres.

Les nombres, à base dix, identifient un ordre céleste absolu celui des archanges ou des intelligences chérubiniques. Les 28 lettres identifient des puissances subordonnées lui permettant d'imposer son règne sur terre à l'aide de cohortes de messagers angéliques. Elles sont en rapport avec les maisons lunaires, multiples elles-mêmes du nombre des éléments (quatre) et de celui des sphères planétaires (sept).

Effectivement des carrés magiques ont été élaborés qui correspondent graphiquement, mystérieusement, à des noms d'Allah ${ }^{36}$ et servent à identifier une puissance divine au nom de laquelle une opération magique est accomplie. Ils prolongent dans le temps, sous une autre forme, l'effet de la nomination verbale d'une même puissance, mais ne paraissent pas en mesure de la remplacer intégralement. Quoi

35 Voir notamment, à ce sujet, l'article de Pierre Lory, 1985: 93 et 94.

${ }^{36}$ Al Bûni $\left(12^{\circ}-13^{\circ}\right.$ siècle) s'en est fait le spécialiste et en présente un grand nombre dans son ouvrage intitulé Sams al ma'ârif ("Le soleil des connaissances"). Constant Hamès y fait ci-dessous référence dans une partie de son article. 
qu'il en soit, des paroles ne manquent jamais, en effet, d'être prononcées lors de la confection rituelle du moindre talisman.

Ainsi l'objet tend à comporter un élément évocateur de la puissance visée et, de son côté, la formule magique qui le complète un élément évocateur du "souffle" en affinité avec elle. Cela s'accorde avec une conception de l'univers en vertu de laquelle tous ses principes, forcés à l'unité, se trouvent représentés d'une certaine manière à chacun de ses niveaux tout effet potentiellement dans sa cause, et toute cause causalement dans son effet.

Nous n'avons jusqu'à présent prêté attention aux objets qu'en vertu de propriétés naturelles et symboliques qui leur sont inhérentes. Il nous reste à mentionner que certains autres peuvent être exploités dans le même but en raison d'empreintes spirituelles leur ayant été communiquées après coup. Tel est le cas de ceux qui ont servi d'instrument ou d'intermédiaire dans la production d'un événement important, par exemple une victoire à la guerre a la suite d'une panique provoquée chez l'ennemi par un curieux incident. Tel est surtout le cas de ceux qui ont été spontanément investis par des esprits indépendants ou par des divinités s'imposant aux hommes par l'intermédiaire de leurs ancêtres à l'occasion de chaque cérémonie du culte, ils se retrouvent affectés par les effluves de leurs occupants. Bien qu'ils n'aient été a l'origine que de simples pierres, accidents de terrain, images innocentes, ustensiles, animaux ou corps humains ne présentant aucune affinité prononcée avec les forces déployées par ceux-ci, ils n'en sont pas moins devenus capables de les symboliser.

Il en résulte que tout autel d'esprit de la nature ou de divinité révélée à ses adorateurs et tenue en relation avec eux par des âmes d'ancêtres ${ }^{37}$ peut être fétichisé et servir de moyen d'accès direct à la source surnaturelle de sa puissance. Pour peu que les représentants défunts de la tradition veuillent bien que les vivants en tirent profit à leur propre gré, et parfois même les y encouragent, il remplira à

${ }^{37}$ Une telle divinité correspond à la notion évhé de $\log b i$ vodu (divinité ancestrale) par opposition à celle de vodu tout court (divinité fétiche) qui se laisse acheter et peut être communiqué à l'extérieur du groupe de parenté, y compris à des étrangers. 
l'occasion une fonction de fétiche. Il peut alors arriver, comme j'ai pu le constater, que l'adepte d'un vodu contraigne en féticheur son vodu à frapper quelqu'un contre lequel il est violemment irrite, mais en soit bientôt châtié par le même vodu à l'instigation d'âmes défuntes d'anciens chefs de culte se réservant de surveiller l'usage qui en est fait et ayant été indignés par sa conduite.

Compte tenu de toutes les équivalences mentionnées, nous pouvons nous attendre à découvrir un fétichisme analogue à celui de l'Afrique noire, entremêlé avec des pratiques considérées comme plus spécifiquement religieuses car soumises aux autorités traditionnelles, dans toutes les civilisations ayant approuvé une mise à profit de la puissance des divinités.

Il ne saurait évidemment fleurir que chez des populations réfractaires à une conception du salut n'entrainnant que mépris pour les affaires terrestres. Ceux qui s'y intéressent valorisent la création et la participation de l'homme à son achèvement, associent étroitement de ce fait mystique, sainteté et pouvoirs, et sont préparés à considérer l'exercice de ces derniers comme une méthode d'enrichissement spirituel aussi bien que d'apaisement des appétits vitaux.

Il n'est pas caractérisé par une adhésion irrationnelle à un certain genre d'objet, mais par un mode de relation singulier et résolu avec des objets.

Pour l'étudier dans toute son ampleur, en tant qu'il nous concerne également et traverse nos propres sociétés, il serait regrettable de le limiter aux aspects qu'il revêt tant au Togo que dans la plupart des autres pays d'Afrique noire. Ceux qui s'y adonnent exemplairement dans ce continent ne font là que spécifier à leur manière un mode universel d'accession à des puissances sacrées.

\author{
A. de Surgy \\ URA 221, EPHE-CNRS
}




\section{Références bibliographiques}

Adohane, Taoufik

1991 "Place et statut de l'objet dans la pratique médicale au Maroc" Nouvelle Revue d'Ethnopsychiatrie, 16, 55-73.

Boston, John

1971 "Medecines and fetishes in Igala", Africa, 41, 3, 200-207.

Brunner, Hélène

1990 "L'image divine dans le culte âgamique de Shiva. Rapport entre l'image mentale et le support concret du culte", dans L'image divine. Culte et méditation dans l'hindouisme, A. Padoux éd., Paris, éd. du CNRS, 9-29.

Cissé, Youssouf

1973 "Signes graphiques, représentations, concepts et tests relatifs à la personne chez les Malinké et les Bambara du Mali", dans La notion de personne en Afrique Noire, Paris, C.N.R.S., 131-179.

Freud, Sigmund

Article sur le fétichisme repris dans "Objets du fétichisme", Nouvelle Revue de psychanalyse, 1970, 2, 24.

Gilli, Bruno

1987 Heviesso et le bon ordre du monde, Lomé, éd. Haho.

Gupta, Sanjukta

1989 "The Pāñcarātra Attitude to Mantra" dans Mantra, H. P. Alper ed., New-York, S.U.N.Y. Press, 224-248.

Hamès, Constant

1987 "Taktub ou la magie de l'écriture islamique. Textes soninké à usage magique", Arabica, XXXIV, 305-325.

Lory, Pierre

1985 "La science des lettres en terre d'islam", Cahiers de l'Université Saint-Jean de Jérusalem, 11, 89-101.

Padoux, André éd.

1990 L'image divine, culte et méditation dans l'hindouisme, Paris, Ed. du CNRS 
Padoux, André

1990 "Image mentale, méditation et culte dans la Shrîvidyâ", dans L'image divine. Culte et méditation dans l'hindouisme, A. Padoux éd., Paris, Ed. du CNRS, 89-98.

Surgy, Albert de

1990 "Bo et vodu protecteurs du sud-Togo", Nouvelle Revue d'Ethnopsychiatrie, 16, 77-100.

Verbeke, Gérard

1945 L'évolution de la théorie du pneuma, du stoïcisme à Saint Augustin, Paris, Desclée de Brouwer. 